\title{
ON $\mathfrak{F}$-NORMALIZERS
}

\author{
TI YEN
}

ABSTRACT. The concept of system normalizers of a finite solvable group is generalized to any saturated formation $\mathfrak{F}$. Given a Sylow system $\Sigma$ of the finite solvable group $G$ and an $\mathfrak{F}$-projector $V$ of $G$ reducing $\Sigma$, a sequence $\left\{H_{i}\right\}$ of subgroups $H_{i}$, is constructed such that

(i) each $H_{i}$ reduces $\Sigma$, and

(ii) the $\mathfrak{F}$-normalizers of $\Sigma \cap H_{i}$ in $H_{i}$ converge to $V$.

The concept of system normalizers has been extended by Carter and Hawkes [4] to any local formation $\mathfrak{F}=\{\mathfrak{F}(p)\}$, where $\mathfrak{F}(p) \subseteq \mathfrak{F}$ is a nonempty formation for each prime $p$. Using an idea of Fischer $[5,62-68]$, we give here an equivalent definition of $\mathfrak{F}$-normalizers which is valid for all integrated (i.e., $\mathfrak{F}(p) \subseteq \mathfrak{F}$ ) local formation. Then we construct a sequence of $\mathfrak{F}$-normalizers which converges to an $\mathfrak{F}$-projector. The $\mathfrak{F}$-projector is a name suggested by Gaschütz for what he originally called an $\mathfrak{F}$-covering subgroup [6].

All groups considered are finite solvable. The notations and terminologies are standard. Given a group $G$ and a formation $\mathfrak{F}, F(G)$ and $\Phi(G)$ denote respectively the Fitting subgroup and the Frattini subgroup of $G$, and $G(\mathfrak{F})$ is the least normal subgroup of $G$ such that $G / G(\mathfrak{F}) \in \mathfrak{F}$. If a subgroup $M$ of $G$ reduces a Sylow system $\Sigma$ of $G$, then $\Sigma \cap M$ denotes the Sylow system of $M$ obtained by intersecting $M$ with the members of $\Sigma$ and $N_{G}(\Sigma \cap M)$ is the relative system normalizer. $\mathfrak{R}$ denotes the formation of all nilpotent groups, and $\mathfrak{N F}$ is the formation of groups which are extensions of nilpotent groups by groups in $\mathfrak{F}$.

1. $\mathfrak{F}$-normalizers. Let $\mathfrak{F}=\{\mathfrak{F}(p)\}$ be an integrated local formation.

Definitions [4]. A $p$-chief factor $H / K$ of a group $G$ is $\mathfrak{F}$-central of $G / C_{G}(H / K) \in \mathfrak{F}(p)$; otherwise, it is $\mathfrak{F}$-eccentric. A maximal subgroup $M$ of $G$ is $\mathfrak{F}$-normal if $M /$ Core $(M) \in \mathfrak{F}(p)$, where $p$ is the prime dividing $[G: M]$; otherwise, $M$ is $\mathfrak{F}$-abnormal.

We need the following results from [4] and [8]:

(A) [4, Lemma 2.3]. A maximal subgroup of $G$ is $\mathfrak{F}$-normal if and only if it complements an $\mathfrak{F}$-central chief factor of $G$.

Received by the editors October 1, 1969.

AMS subject classifications. Primary 2040; Secondary 2025, 2043.

Key words and phrases. Solvable group, formation, system normalizer, $\mathfrak{F}$-normalizer. 
(B) [4, Lemma 4.4]. $G \in \mathfrak{F}$ if and only if every minimal normal subgroup of $G / \Phi(G)$ is $\mathfrak{F}$-central.

(C) [4, Theorem 5.8]. Let $G \in \mathfrak{N F}$ and $H$ be a subgroup of $G$ such that $H \in \mathfrak{F}$ and $G=F(G) \cdot H$. Then $H$ is contained in some $\mathfrak{F}$-projector of $G$.

(D) $[8$, Satz 2.1]. Let $V$ be an $\mathfrak{F}$-projector of a group $G$. Then $N M \cap V=(N \cap V)(M \cap V)$, for any normal subgroups $N, M$ of $G$.

Lемма 1.1. (a) $G(\mathfrak{F})$ covers all $\mathfrak{F}$-eccentric chief factors of a group $G$.

(b) $M(\mathfrak{F}) \subseteq G(\mathfrak{F})$ for any $\mathfrak{F}$-abnormal subgroup $M$ of $G$.

Proof. (a) It is sufficient to show that $G(\mathfrak{F})$ covers the $\mathfrak{F}$-eccentric minimal normal subgroups. Let $A$ be an $\mathfrak{F}$-eccentric minimal normal $p$-subgroup, and let $V$ be an $\mathfrak{F}$-projector of the group $G$. Then

$$
\begin{aligned}
A & =A \cap A \cdot G(\mathfrak{F}) \cap V \cdot G(\mathfrak{F}) \\
& =A \cap[A \cdot G(\mathfrak{F}) \cap V] \cdot G(\mathfrak{F}) \\
& =A \cap[(A \cap V)(G(\mathfrak{F}) \cap V)] \cdot G(\mathfrak{F}) \quad(\text { by }(\mathrm{D})) \\
& =(A \cap V)(A \cap G(\mathfrak{F})) .
\end{aligned}
$$

If $A \cap G(\mathfrak{F})=1$, then $A$ centralizes $G(\mathfrak{F})$ and is a minimal normal $p$-subgroup of $V$. It follows that $G / C_{G}(A) \cong V / C_{V}(A) \in \mathfrak{F}(p)$. This is a contradiction to $A$ being $\mathfrak{F}$-eccentric.

(b) Let $K=$ Core $(M)$ and let $H / K$ be the chief factor complementing $M$. Then $H=(H \cap G(\mathfrak{F})) \cdot K, \quad G=(H \cap G(\mathfrak{F})) \cdot M$, and $G(\mathfrak{F})$ $=(H \cap G(\mathfrak{F})) \cdot M(\mathfrak{F})$.

LemMa 1.2. Let $H$ be a normal subgroup of a group $G$. Then $N_{G}(\Sigma \cap H)$ reduces $\Sigma$, for any Sylow system $\Sigma$ of $G$ [7, Theorem 4.1].

Proof. We have $G=H \cdot N_{G}(\Sigma \cap H)$. Let $\Sigma_{0}$ be a Sylow system of $N_{G}(\Sigma \cap H)$. Then $(\Sigma \cap H) \Sigma_{0}=\left\{\left(S_{p} \cap H\right) T_{p} \mid S_{p} \in \Sigma, T_{p} \in \Sigma_{0}\right\}$ is a Sylow system of $G$. Hence $(\Sigma \cap H) \Sigma_{0}=\Sigma^{x}$, for some $x \in G$. Since $\Sigma \cap H$ $=(\Sigma \cap H) \Sigma_{0} \cap H=\Sigma^{x} \cap H=(\Sigma \cap H)^{x}, x \in N_{G}(\Sigma \cap H)$. Therefore $\Sigma$ reduces into $N_{G}(\Sigma \cap H)$.

Notations. $\mathfrak{D}(\Sigma)$ denotes the set of $\mathfrak{F}$-projectors of $N_{G}(\Sigma \cap G(\mathfrak{F}))$ which reduce $\Sigma$. If $H$ reduces $\Sigma$ then $\mathfrak{D}(\Sigma \cap H)$ is the set of $\mathfrak{F}$-projectors of $N_{H}(\Sigma \cap H(\mathfrak{F}))$ which reduce $\Sigma$.

Since $N_{G}(\Sigma \cap G(\mathfrak{F}))$ reduces $\Sigma, \mathfrak{D}(\Sigma)$ is not empty. The aim of this section is to show that $\mathfrak{D}(\Sigma)$ consists of only one element $D(\Sigma)$. Its proof, through a series of lemmas, follows closely the development in [5].

Lemma 1.3. (a) If $G \in \mathfrak{N F}$ then $G=N_{G}(\Sigma \cap G(\mathfrak{F}))$, for any Sylow system $\Sigma$ of $G$. Therefore the elements of $\mathfrak{D}(\Sigma)$ are $\mathfrak{F}$-projectors of $G$. 
(b) For any epimorphism $\alpha$ of $G, \mathfrak{D}(\Sigma)^{\alpha} \subseteq \mathfrak{D}\left(\Sigma^{\alpha}\right)$.

Proof. Since $G(\mathfrak{F})$ is a nilpotent normal subgroup, (a) is valid. (b) also holds, because each step in the construction of $\mathfrak{D}(\Sigma)$ is invariant under epimorphisms.

Lemma 1.4. Let $\Sigma$ be a Sylow system of a group $G$, and let $H$ be a normal subgroup containing $G(\mathfrak{F})$. Then the following statements hold:

(a) $N_{G}(\Sigma \cap H) \in \mathfrak{R F}$. Therefore the elements of $\mathfrak{D}\left(\Sigma \cap N_{G}(\Sigma \cap H)\right)$ are $\mathfrak{F}$-projectors of $N_{G}(\Sigma \cap H)$.

(b) Each element of $\mathfrak{D}\left(\Sigma \cap N_{G}(\Sigma \cap H)\right)$ is contained in an element of $\mathfrak{D}(\Sigma)$.

Proof. (a) Since $G=H \cdot N_{G}(\Sigma \cap H)$ and $H \supseteq G(\mathfrak{F})$,

$$
N_{G}(\Sigma \cap H) / N_{H}(\Sigma \cap H) \cong G / H
$$

is an epimorphic image of $G / G(\mathfrak{F})$. Therefore $N_{G}(\Sigma \cap H) \in \mathfrak{R} \mathfrak{F}$, and the elements of $\mathfrak{D}\left(\Sigma \cap N_{G}(\Sigma \cap H)\right)$ are $\mathfrak{F}$-projectors of $N_{G}(\Sigma \cap H)$, as shown in Lemma 1.3 (a).

(b) We use induction on the order $|G|$. Let $G$ be a minimal counterexample and let $H$ be of minimal index [H:G(₹)] for which the lemma fails. Let $H / K$ be a chief factor of $G / G(\mathfrak{F})$. Then each element of $\mathfrak{D}\left(\Sigma \cap N_{G}(\Sigma \cap K)\right)$ is contained in an element of $\mathfrak{D}(\Sigma)$.

From

$$
G=K \cdot N_{G}(\Sigma \cap K)=H \cdot N_{G}(\Sigma \cap K),
$$

we have $N_{G}(\Sigma \cap K) / N_{H}(\Sigma \cap K) \cong G / H \in \mathcal{F}$. Therefore the pair $N_{G}(\Sigma \cap K)$ and $N_{H}(\Sigma \cap K)$ satisfies the hypothesis of the lemma. Since $H=K \cdot N_{H}(\Sigma \cap K)$ and $N_{G}(\Sigma \cap H)$ normalizes $N_{H}(\Sigma \cap K)$, the relative system normalizer of $\Sigma \cap N_{H}(\Sigma \cap K)$ in $N_{G}(\Sigma \cap K)$ is $N_{G}(\Sigma \cap H)$. If $N_{G}(\Sigma \cap K) \neq G$ then, by induction, each element of $\mathfrak{D}\left(\Sigma \cap N_{G}(\Sigma \cap H)\right)$ is contained in an element of $\mathfrak{D}\left(\Sigma \cap N_{G}(\Sigma \cap K)\right)$. Hence each element of $\mathfrak{D}\left(\Sigma \cap N_{G}(\Sigma \cap H)\right)$ is contained in an element of $\mathfrak{D}(\Sigma)$.

If $N_{G}(\Sigma \cap K)=G$ then $K$ is nilpotent. Thus $G \in \mathfrak{R F}$. Let $p$ be the prime dividing $|H / K|$. Then $H=\left(S_{p} \cap H\right) K$, where $S_{p}$ is the Sylow p-subgroup in $\Sigma$. Then $N_{G}(\Sigma \cap H)=N_{G}\left(S_{p} \cap H\right) \supseteq S_{p} \cap H$. Hence

$$
G=H \cdot N_{G}(\Sigma \cap H)=K \cdot N_{G}(\Sigma \cap H)=K E,
$$

where

$$
E \in \mathfrak{D}\left(\Sigma \cap N_{G}(\Sigma \cap H)\right) .
$$

By (C), $E$ is contained in an $\varsubsetneqq$-projector $D$ of $G$. Since $K$ is a nilpotent 
normal subgroup, $D \cap K$ reduces any Sylow system of $G$. Therefore $D=(D \cap K) E$ reduces $\Sigma$, by [2, Lemma 2.7]. This completes the proof.

LeMma 1.5. Let $M$ be an $\mathfrak{F}$-abnormal subgroup of $G$ which reduces $a$ Sylow system $\Sigma$. Then each element of $\mathfrak{D}(\Sigma)$ is contained in an element of $\mathfrak{D}(\Sigma \cap M)$.

Proof. Let $G$ be a minimal counterexample.

(1) $\mathfrak{D}(\Sigma) \subset M$. If Core $(M) \neq 1$ then $G /$ Core $(M)$ satisfies the lemma. Therefore $\mathfrak{D}(\Sigma) \subset M$ if Core $(M) \neq 1$.

Suppose Core $(M)=1$. Then there is a self-centralizing minimal normal $p$-subgroup $A$ which complements $M$. We have $A \subseteq G(\mathfrak{F})$ and $M(\mathfrak{F})=M \cap G(\mathfrak{F})$, by (A) and Lemma 1.1. If $M(\mathfrak{F})=1$ then $M$ is an F-projector of $G=N_{G}(\Sigma \cap G(\mathfrak{F}))$ [9]. Suppose that $\Sigma$ also reduces into $M^{a}$, where $1 \neq a \in A$. Then the Sylow $p$-complement $S^{p}$ of $\Sigma$ is contained in $M$ and $M^{a}$. Hence $\left[S^{p}, a\right] \subset M^{a} \cap A=1$, and $a$ centralizes $O_{p^{\prime}}(M)$. This contradicts the assumption that $A$ is self-centralizing. Hence $M$ is the unique $\mathfrak{F}$-projector reducing $\Sigma$. Suppose $M(\mathfrak{F}) \neq 1$. We show that $N_{G}(\Sigma \cap G(\mathfrak{F})) \subseteq M$. Suppose $a m \in N_{G}(\Sigma \cap G(\mathfrak{F}))$, where $1 \neq a \in A$ and $m \in M$. Since $M \supseteq S^{p}$ and $S^{p} \cap G(\mathfrak{F})=\left(S^{p} \cap G(\mathfrak{F})\right)^{a m}$, we have $\left[S^{p} \cap G(\mathfrak{F}), a\right] \subseteq A \cap M=1$. Then $a \in C_{A}\left(O_{p^{\prime}}(M(\mathfrak{F}))\right)$. Hence $A=C_{A}\left(O_{p^{\prime}}(M(\mathfrak{F}))\right)$. This contradicts the assumption that Core $(M)=1$.

(2) Each element of $\mathfrak{D}(\Sigma)$ is contained in an element of $\mathfrak{D}\left(\Sigma \cap N_{M}(\Sigma \cap G(\mathfrak{F}) \cap M)\right.$. Let $D \in \mathfrak{D}(\Sigma)$. We have

$$
\begin{aligned}
D & \subseteq N_{G}(\Sigma \cap G(\mathfrak{F})) \cap M=N_{M}(\Sigma \cap G(\mathfrak{F})) \\
& \subseteq N_{M}(\Sigma \cap G(\mathfrak{F}) \cap M), \\
G & =G(\mathfrak{F}) \cdot D,
\end{aligned}
$$

and

$$
\begin{aligned}
N_{M}(\Sigma \cap G(\mathfrak{F}) \cap M) & =\left[N_{M}(\Sigma \cap G(\mathfrak{F}) \cap M) \cap G(\mathfrak{F})\right] \cdot D \\
& =N_{G(\mathfrak{F}) \cap M}(\Sigma \cap G(\mathfrak{F}) \cap M) \cdot D .
\end{aligned}
$$

By (C), $D$ is contained in an $\mathfrak{F}$-projector $E$ of $N_{M}(\Sigma \cap G(\mathfrak{F}) \cap M)$. Then $E=\left[E \cap N_{G(\mathfrak{F}) \cap_{M}}(\Sigma \cap G(\mathfrak{F}) \cap M)\right] \cdot D$ reduces $\Sigma$, by [2, Lemma 2.7]. Therefore $E \in \mathfrak{D}\left(\Sigma \cap N_{M}(\Sigma \cap G(\mathfrak{F}) \cap M)\right)$.

(3) Each element of $\mathfrak{D}\left(\Sigma \cap N_{M}(\Sigma \cap G(\mathfrak{F}) \cap M)\right)$ is contained in an element of $\mathfrak{D}(\Sigma \cap M)$. The pair $M$ and $G(\mathfrak{F}) \cap M$ satisfies the hypothesis of Lemma 1.4 (b). Therefore (3) is valid. This completes the proof.

THEOREM 1.1. $\mathfrak{D}(\Sigma)$ contains only one element. 
Proof. We use induction on $|G|$. Let $G$ be a minimal counterexample. Then $G \notin \mathfrak{F}$ and there exists an $\mathfrak{F}$-abnormal subgroup $M$, which reduces $\Sigma$ and complements a $p$-chief factor $A / \Phi(G)$. Let $P$ be the Sylow $p$-subgroup of $A \cap G(\Im)$. Since $A$ is nilpotent, $P$ is a normal subgroup supplementing $M$. Since $G(\mathfrak{F})=P \cdot M(\mathfrak{F})$, $N_{M}(\Sigma \cap M(\mathfrak{F})) \subseteq N_{M}(\Sigma \cap G(\mathfrak{F})) \subseteq N_{G}(\Sigma \cap G(\mathfrak{F}))$. By Lemma 1.5, each element of $\mathfrak{D}(\Sigma)$ is contained in an element of $\mathfrak{D}(\Sigma \cap M)$. Since $N_{M}(\Sigma \cap M(\mathfrak{F})) \subseteq N_{G}(\Sigma \cap G(\mathfrak{F}))$, each element of $\mathfrak{D}(\Sigma)$ is an element of $\mathfrak{D}(\Sigma \cap M)$. By induction, $\mathfrak{D}(\Sigma \cap M)$ has only one element. Therefore $\mathfrak{D}(\Sigma)$ has only one element.

Definition. The unique element $D(\Sigma)=D$ of $\mathfrak{D}(\Sigma)$ is called the F-normalizer of the Sylow system $\mathbf{\Sigma}$.

We may restate Lemma 1.5 and the proof of Theorem 1.1 as follows:

TheOREM 1.2. Let $M$ be an $\mathfrak{F}$-abnormal subgroup reducing a Sylow system $\Sigma$ of $G$. Then $D(\Sigma \cap M) \supseteq D(\Sigma)$. If, in addition, $M$ complements an $\mathfrak{F}$-eccentric minimal normal subgroup of $G / \Phi(G)$, then $D(\Sigma \cap M)$ $=D(\Sigma)$.

REMARK. If $\mathfrak{F}$ contains the formation $\mathfrak{N}$ of nilpotent groups, then our definition of $\mathfrak{F}$-normalizer coincides with the $\mathfrak{F}$-normalizer of Carter and Hawkes.

Proof. Let $C_{p}$ be the intersection of all $C_{G}(H / K)$, where $H / K$ runs over all $\mathfrak{f}$-central $p$-chief factors. Put $D_{0}(\Sigma)=\bigcap_{p} N_{G}\left(S^{p} \cap C_{p}\right)$, where $S^{p}$ is the Sylow $p$-complement in the Sylow system $\Sigma . D_{0}(\Sigma)$ is the F-normalizer of Carter and Hawkes. If a maximal subgroup $M$ reduces $\Sigma$ and complements an $\mathfrak{F}$-eccentric minimal normal subgroup of $G / \Phi(G)$, then [4, Lemma 4.6] $D_{0}(\Sigma)=D_{0}(\Sigma \cap M)$. Hence, by induction and Theorem 1.2, $D(\Sigma)=D(\Sigma \cap M)=D_{0}(\Sigma \cap M)=D_{0}(\Sigma)$.

The following theorem is [5, Theorem 8.5$]$. Fischer's proof, which we reproduce here, does not require $\mathfrak{F} \supseteq \mathfrak{N}$.

TheOREM 1.3. $D=D(\Sigma)$ covers all $\mathfrak{F}$-central chief factors and avoids all $\mathfrak{F}$-eccentric chief factors of $G$.

Proof. By Lemma 1.3 (b), it is sufficient to show that $D$ covers all $\mathfrak{F}$-central minimal normal subgroups of $G$ and avoids all $\mathfrak{F}$-eccentric ones. Let $A$ be an $₹$-central minimal normal $p$-subgroup of $G$. Since $G / C_{G}(A) \in \mathfrak{F}(p) \subseteq \mathfrak{F}, A$ centralizes $G(\mathfrak{F})$. Consequently $A \subseteq N_{G}(\Sigma \cap G(\mathfrak{F}))$, and $A$ is a minimal normal $p$-subgroup of $A D$. As $A D / C_{A D}(A) \cong G / C_{G}(A) \in \mathfrak{F}(p)$, we have $A D \in \mathfrak{F}$. Since $D$ is an F-projector of $N_{G}(\Sigma \cap G(\mathfrak{F})), A D=D$. 
Let $A$ be an $\mathfrak{F}$-eccentric minimal normal $p$-subgroup of $G$. We have $A \subseteq G(\mathfrak{F})$ and $A \cap D \subseteq G(\mathfrak{F}) \cap N_{G}(\Sigma \cap G(\mathfrak{F}))=N_{G(\mathfrak{F})}(\Sigma \cap G(\mathfrak{F}))$. Suppose $A \cap D \neq 1$. As a system normalizer covers the central chief factors and avoids the eccentric ones, $A \cap D \subseteq Z(G(\mathfrak{F}))$. Then $A \cap D$ is a normal subgroup. Consequently $A=A \cap D$ is also a minimal normal $p$-subgroup of $D$. Then $G / C_{G}(A) \cong D / C_{D}(A) \in \mathfrak{F}(p)$. This contradicts the assumption that $A$ is $\mathfrak{F}$-eccentric.

REMARK. A normal subgroup $N$ of a group $G$ is called $\mathfrak{F}$-hypercentral

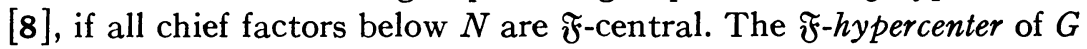
is the maximal $\mathfrak{F}$-hypercentral subgroup of $G$. Theorem 1.3 yields the usual characterization of $\mathfrak{F}$-hypercenter as the intersection of all F-normalizers.

\section{A convergent sequence.}

Lemma 2.1. Let $\Sigma$ be a Sylow system of a group $G$, and $D=D(\Sigma)$. Then the following statements hold:

(a) If an $\mathfrak{F}$-projector $V$ of $G$ reduces $\Sigma$, then $V \supseteq D$.

(b) $D(\Sigma \cap G(\mathfrak{N F}) \cdot D) \supseteq D$.

Proof. (a) Suppose there exists a maximal subgroup $M$ which contains $V$ and reduces $\Sigma$. By [4, Lemma 5.1], $M$ is $₹$-abnormal. Then $D(\Sigma \cap M) \supseteq D$ by Theorem 1.2, and $V \supseteq D(\Sigma \cap M)$ by induction. It remains to produce such a maximal subgroup. Let $A$ be a minimal normal subgroup of $G$. If $G=A V$, then $V$ is maximal. If $G \neq A V$ then, by induction $G / A$ has a maximal subgroup $M / A$ which reduces $\Sigma A / A$ and contains $A V / A$. Then $M$ reduces $\Sigma$ and contains $V$.

(b) Since $G / G(\mathfrak{N F}) \in \mathfrak{N F}, G(\mathfrak{N F}) \cdot D / G(\mathfrak{N F})$ is an $\mathfrak{F}$-projector of $G / G(\mathfrak{N F})$ by Lemma 1.3 . From the above paragraph, there exists an $\mathfrak{F}$-abnormal subgroup $M_{1}$, which reduces $\Sigma$ and contains $G(\mathfrak{N} \mathfrak{F}) \cdot D$. Repeat this process, we get a chain of subgroups $G=M_{0} \supset M_{1} \supset \ldots$ $\supset M_{r}=G(\mathfrak{N} \mathfrak{F}) \cdot D$, where each $M_{i}$ is $\mathfrak{F}$-abnormal in $M_{i-1}$ and reduces $\Sigma, 1 \leqq i \leqq r$. Apply Theorem 1.2 to this chain and get $D(\Sigma \cap G(\mathfrak{N F}) \cdot D)$ $\supseteq D$.

Given a group $G$ and a Sylow system $\Sigma$ of $G$, we construct two sequences $\left\{H_{i}\right\}$ and $\left\{D_{i}\right\}$ as follows:

$$
H_{0}=G, D_{0}=D\left(\Sigma \cap H_{0}\right) \text {, and } H_{i}=H_{i-1}(\mathfrak{N F}) \cdot D_{i-1}, D_{i}=D\left(\Sigma \cap H_{i}\right) \text {, }
$$

for $i \geqq 1$. We have $H_{0} \supseteq H_{1} \supseteq H_{2} \supseteq \ldots$, and $D_{0} \subseteq D_{1} \subseteq D_{2} \subseteq \ldots$ by Lemma 2.1 (b). Since $H_{i}(\mathfrak{N F})$ is the smallest normal subgroup of $H_{i}(\mathfrak{F})$ with a nilpotent factor $H_{i}(\mathfrak{F}) / H_{i}(\mathfrak{N} \mathfrak{F})$, the $H$-sequence terminates at $H_{r} \in \mathfrak{F}$, if $H_{r-1} \in \mathfrak{N F}$. Then the $D$-sequence terminates at $D_{r-1}=H_{r}$. 
TheOREм 2.1. $D_{r-1}=H_{r}$ is an $\mathfrak{F}$-projector of $G$ which reduces $\Sigma$.

Proof. Since $H_{i} / H_{i}(\mathfrak{N F}) \in \mathfrak{N} F, H_{i+1}$ contains an $\mathfrak{F}$-projector of $H_{i}$ by Lemma 1.3 (a). Therefore the $\mathfrak{F}$-projector of $H_{i+1}$ are the $\mathfrak{F}$-projectors of $H_{i}$. Thus $H_{r}$ is an $\mathfrak{F}$-projector of $G$. $H_{r}$ reduces $\Sigma$ by the construction of the sequence.

Corollary 2.1. Any Sylow system $\Sigma$ reduces into exactly one $\mathfrak{F}$-projector [1, Theorem 6].

Proof. Suppose that $\Sigma$ reduces into an $\mathfrak{F}$-projector $V$. Then $V \supseteq D_{0}$ by Lemma 2.1 (a). Since $H_{1} / H_{0}(\mathfrak{N F})$ is an $\mathfrak{F}$-projector of $H_{0} / H_{0}(\mathfrak{N F})$, we have $D_{0} \subseteq V \subseteq H_{1}$. Suppose that $D_{i-1} \subseteq V \subseteq H_{i}$. As $V \subseteq H_{i}$ and reduces $\Sigma$, we get $V \supseteq D_{i}$. Similarly, since $H_{i+1} / H_{i}(\mathfrak{N F})$ is an $\mathfrak{F}$-projector of $H_{i} / H_{i}(\mathfrak{R F})$, we have $V \subseteq H_{i+1}$. Therefore $D_{r-1}=V=H_{r}$ is the unique $\mathfrak{F}$-projector reducing $\boldsymbol{\Sigma}$.

CoROLLARY 2.2. If $G \in \mathfrak{N N F}$ then each $\mathfrak{F}$-normalizer is contained in only one $\mathfrak{F}$-projector of $G[4$, Theorem 5.9].

Proof. Let $G \in \mathfrak{N N F}$. Then $G(\mathfrak{F}) \in \mathfrak{R}, H_{1}=G(\mathfrak{N F}) \cdot D_{0} \in \mathfrak{R F}$, and $H_{2}=D_{1} \in \mathfrak{F}$. Suppose that $D_{0}$ is contained in an $\mathfrak{F}$-projector $V$. Then $V$ is contained in $G(\mathfrak{N F}) \cdot D_{0}$. Since $G(\mathfrak{N F})$ is a nilpotent normal subgroup of $H_{1}, \Sigma \cap H_{1}$ reduces into $V \cap G(\mathfrak{N F})$. Hence $\Sigma$ reduces into $[V \cap G(\mathfrak{N F})] \cdot D_{0}=V$, by $\left[2\right.$, Lemma 2.7]. Then $V=D_{1}$, by Corollary 2.1.

CoRollaRY 2.3. Suppose $G \in \mathfrak{R N F}$. Let $V$ be an $\mathfrak{F}$-projector containing the F-normalizer $D$ of a Sylow system $\Sigma$ of $G$. If $V$ is self-centralizing, then $V \supseteq K$, for every subgroup $K$ such that $D$ is subnormal in $K$ [4, Theorem 5.10].

Proof. Let $D=K_{0} \subset K_{1} \subset \cdots \subset K_{8}=K$ be a subnormal series joining $D$ and $K$. Suppose $K_{i} \subseteq V$. Since $D \subseteq K_{i}=K_{i}^{x}$, for any $x \in K_{i+1}$, $D \subseteq V^{x}$. By Corollary 2.2, $V=V^{x}$. If $V$ is self-normalizing then $x \in V$. Therefore $K_{i+1} \subset V$. Hence $K \subseteq V$.

\section{REFERENCES}

1. J. L. Alperin, System normalizers and Carter subgroups, J. Algebra 1 (1964), 355-366. MR 30 \#3911.

2. R. W. Carter, On a class of finite soluble groups, Proc. London Math. Soc. (3) 9 (1959), 623-640. MR 22 \#5677.

3. - Nilpotent self-normalizing subgroups and system normalizers, Proc. London Math. Soc. (3) 12 (1962), 535-563. MR 25 \#3988.

4. Roger Carter and Trevor Hawkes, The $\mathfrak{F}$-normalizers of a finite soluble group, J. Algebra 5 (1967), 175-202. MR 34 \#5914. 
5. Bernd Fischer, Classes of conjugate subgroups in finite solvable groups, Lecture Note, Yale University, New Haven, Conn., 1966.

6. Wolfgang Gaschütz, Zur Theorie der endlichen auflösbaren Gruppen, Math. Z. 80 (1962/63), 300-305. MR 31 \#3505.

7. P. Hall, On the system normalizers of a soluble group, Proc. London Math. Soc. (2) 43 (1937), 507-528.

8. Bertram Huppert, Zur Theorie der Formationen, Arch. Math. 19 (1968), 561574.

9. E. Shult, $A$ note on splitting in solvable groups, Proc. Amer. Math. Soc. 17 (1966), 318-320.

Michigan State University, East Lansing, Michigan 48823 